\title{
Idiopathische Inflammatorische Myopathien: Klinisches Spektrum und Diagnostik
}

Julia Wanschitz

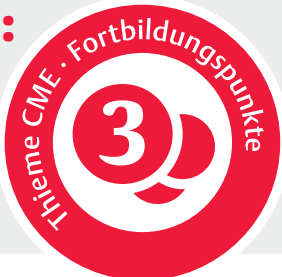

Idiopathische inflammatorische Myopathien (IIM) bzw. Myositiden sind seltene und heterogene, jedoch mit einer hohen Morbidität behaftete Erkrankungen, die eine rasche Diagnose und Therapie erfordern. Die Kernsymptome einer akuten/ subakuten Muskelschwäche und Erhöhung der Creatinkinase können mit verschiedenen Begleitsymptomen einhergehen und müssen im akuten Stadium von einer Rhabdomyolyse abgegrenzt werden. Eine Antikörperdiagnostik trägt wesentlich zur Subklassifikation und Prognoseeinschätzung der Myositiden bei.

\section{Allgemeine Aspekte idiopathischer inflammatorischer Myopathien}

Entzündliche Erkrankungen der Muskulatur führen zu einer Muskelschwäche, welche sich zumeist subakut im Laufe einiger Wochen entwickelt und vorzugsweise proximale und axiale Muskelgruppen in symmetrischer Verteilung befällt. Klinisch imponieren Paresen der Schulter- und Beckengürtelmuskulatur und der axialen Muskulatur.
Virale Infekte (z. B. Influenza A/B, Parainfluenza, Enteroviren) können besonders bei Kindern zu einer benignen, selbstlimitierenden Myositis mit passagerer Muskelschwäche, Wadenschmerzen und CK-Erhöhung führen, die spontan remittiert $[1,2]$. Rezente Berichte weisen auch auf eine SARS-Cov-2-Infektion als möglichen Trigger für eine Myositis hin [3,4]. Bei Patienten mit einer gestörten Immunregulation kann sich ein Autoimmunprozess gegen Antigene der Muskulatur etablie-

\begin{tabular}{|c|c|c|c|}
\hline \multicolumn{4}{|c|}{ ABKÜRZUNGEN } \\
\hline Anti-MDA5 & $\begin{array}{l}\text { Antikörper gegen Melanoma-Diffe- } \\
\text { rentiation-Associated Gene } 5\end{array}$ & IMNM & $\begin{array}{l}\text { immun mediierte nekrotisierende } \\
\text { Myopathie }\end{array}$ \\
\hline ASA & Antisynthetase-Autoantikörper & IVIG & intravenöses Immunglobulin \\
\hline \multirow[t]{2}{*}{ Anti-CCP } & Antikörper gegen zyklisches citrulli- & KG & Kraftgrad \\
\hline & niertes Petid & LDH & Laktatdehydrogenase \\
\hline CK & Creatinkinase & MHC & Major Histocompatibility Complex \\
\hline \multirow[t]{2}{*}{ Anti-cN1a } & Antikörper gegen zytosolische & MAA & Myositis assoziierte Antikörper \\
\hline & 5'-Nucleotidase 1A & MSA & Myositis spezifische Antikörper \\
\hline $\operatorname{cox}$ & Cytochrom-C-Oxidase & MUAP & Muskelaktionspotenzial \\
\hline DM & Dermatomyositis & NK-Zellen & Natural-Killer-Zellen \\
\hline EMG & Nadelelektromyografie & NXP & nucelar Matrix Protein 2 \\
\hline FDG-PET & $\begin{array}{l}\text { Fluordesoxyglukose-Positronenemis- } \\
\text { sionstomografie }\end{array}$ & PCSK9 & $\begin{array}{l}\text { Proproteinkonvertase Subtilisin Kexin } \\
\text { Typ } 9\end{array}$ \\
\hline GOT & Glutamat-Oxalacetat-Transaminase & PEG & endoskopische Gastrostomie \\
\hline GPT & Glutamat-Pyruvat-Transaminase & PM & Polymyositis \\
\hline HLA & Human Leukocyte Antigen & SARS-Cov-2 & severe acute respiratory Syndrome \\
\hline \multirow[t]{2}{*}{ HMGCR } & Hydroxy-3-Methylglutaryl Coenzym & & Coronavirus-2 \\
\hline & A Reduktase & SRP & Signal Recognition Particle \\
\hline IBM & Einschlusskörpermyositis & STIR & Short-Tau Inversion Recovery \\
\hline IIM & $\begin{array}{l}\text { idiopathische inflammatorische } \\
\text { Myopathie }\end{array}$ & TIF-1Y & $\begin{array}{l}\text { transcriptional intermediary Factor } 1 \\
\text { Gamma }\end{array}$ \\
\hline ILD & interstitielle Lungenerkrankung & tRNA & Transfer- Ribonukleinsäure \\
\hline
\end{tabular}


ren und so zu einer fortschreitenden strukturellen Muskelschädigung führen [5].

Die genetische Prädisposition, an einer Myositis zu erkranken wird u.a. vom „Major Histocompatibility Complex“ (MHC) determiniert. So sind bestimmte HLA-Haplotypen mit homogenen Subgruppen der Myositiden assoziiert [6]. Umweltfaktoren wie virale Infekte, Sonnenexposition, Rauchen, Medikamente und toxische Substanzen dürften für die Manifestation der Erkrankung ebenfalls eine Rolle spielen [5]. An der Pathogenese sind sowohl Bestandteile der angeborenen Immunität (z. B. NK-Zellen, Cytokine, Aufregulation von Interferon $y$ ) als auch erworbene Immunmechanismen (z. B. T- und B-Lymphozyten, Antikörper, Komplementsystem) beteiligt [5].

Eine Erhöhung der Creatinkinase (CK) kann, wenngleich für die Art der Muskelschädigung nicht spezifisch, als Maß für den akuten Muskelfaserzerfall oder die Nekrose herangezogen werden [7]. Im chronischen Stadium können die CK-Werte wieder sinken bzw. sich sogar normalisieren (z.B. Dermatomyositis), auch wenn eine Muskelschwäche persistiert.

\section{Cave}

Allerdings schließen eine Creatinkinase < $1000 \mathrm{U} / \mathrm{I}$ und das Fehlen von Muskelschmerzen eine Myositis nicht aus.

Myalgien sind bei einem Teil der PatientInnen vorhanden, jedoch nicht obligat. Respiratorische Probleme können Folge einer Schwäche der respiratorischen Muskulatur oder Ausdruck einer interstitiellen Lungenerkrankung sein. Eine (eventuell subklinische) Myokarditis kann die Skelettmuskelschwäche begleiten [8]. Weitere Organmanifestationen, z. B. der Gelenke, des Gastrointestinaltraktes oder der Niere, sind insbesondere bei Assoziation mit Kollagenosen (Overlap-Syndromen) möglich [9].

Die Differenzialdiagnose einer medikamentös-toxisch oder metabolisch bedingten Rhabdomyolyse (Creatinkinase $>1000 \mathrm{U} / \mathrm{I}$ ) ist besonders für die immun mediierte nekrotisierende Myopathie (IMNM) relevant. Im Gegensatz zu den IMNM ist bei Rhabdomyolyse eine massive Creatinkinase-Erhöhung bedingt durch Muskelfasernekrosen und Zerstörung der Muskelfasermembran unter Flüssigkeitssubstitution binnen einiger Tage rückläufig. Paresen bestehen in der Regel nicht, die Patienten sind jedoch durch Elektrolytentgleisungen und Niereninsuffizienz bis zum akuten Nierenversagen gefährdet und benötigen ein engmaschiges Monitoring bzw. eine intensivmedizinische Behandlung [10].

\section{Merke}

Eine akute/subakute symmetrische proximale/axiale Muskelschwäche und eine deutliche CreatinkinaseErhöhung sind Kernsymptome einer Myositis. Im Gegensatz dazu haben PatientInnen mit einer Rhabdomyolyse (Creatinkinase > $1000 \mathrm{U} / \mathrm{I}$ ) üblicherweise keine Paresen, und die HyperCKämie ist binnen weniger Tage rückläufig.

\section{Krankheitsbilder}

\section{Myositiden mit Hautmanifestationen}

Dermatomyositis (DM)

Die Dermatomyositis ist die häufigste Form einer idiopathischen inflammatorischen Myopathie und bereitet aufgrund der charakteristischen Hauterscheinungen zumeist keine diagnostischen Schwierigkeiten [9]:

- heliotropes Erythem in Gesicht und Dekolletee,

- Lidödeme,

- Gottron'sche Knötchen,

- periunguales Erythem und Teleangiektasien

$(\triangleright$ Abb. 1 )

- kutane Kalzinose

- Alopezie,

- Pannikulitis.

Selten können die Hautmanifestationen der Muskelschwäche jedoch auch nachfolgen, sodass eine Muskelbiopsie für die Diagnose erforderlich sein kann. Die Erkrankung betrifft Kinder (juvenile DM, zumeist im Alter von 5-15 Jahren) [1] oder Erwachsene im mittleren Alter (adulte DM) [9].

Anamnestisch berichten die PatientInnen Probleme bei Tätigkeiten mit erhobenen Armen, beim Treppensteigen oder Aufstehen aus dem Sitzen/der tiefen Hocke etc.; auch die körperliche Ausdauer kann deutlich beeinträchtigt sein.

In der klinischen Untersuchung sind Armabduktion und Hüftbeugung am stärksten betroffen; nicht selten besteht eine axiale Schwäche (Probleme beim Aufrichten aus dem Liegen, Schwäche für die Nackenflexion und -extension). Bei schwerer Krankheitsmanifestation kann das Schlucken beeinträchtigt sein. Augenmuskelparesen gehören nicht zum klinischen Bild einer systemischen Myositis. Muskelatrophien liegen zu Beginn der Erkrankung auch bei ausgeprägter Schwäche nicht vor, können sich aber bei unzureichender Therapie oder fehlendem Therapieansprechen im Verlauf entwickeln.

Die Creatinkinase ist im akuten Stadium auf das 10 - bis 50-Fache des Normalwertes erhöht. Durch die Assoziation mit Myositis-spezifischen bzw. -assoziierten Antikörpern ist die weitere Untergliederung in homogene 
Subtypen möglich [11]. So zeigt eine DMmit einem klassischen Exanthem in sonnenexponierten Arealen und Mi-2-Antikörpern typischerweise ein gutes Ansprechen auf Therapie. Eine Dermatomyositis mit antiTIF-1Y (transcriptional intermediary Factor 1 Gamma) oder anti-NXP (nuclear Matrix Protein 2)-Antikörpern kann einen besonders aggressiven Verlauf nehmen mit

- ausgeprägten Paresen,

- ulzerierenden Hautveränderungen [12] oder

- subkutanen Verkalkungen [13].

Die DM des Erwachsenenalters ist, im Gegensatz zur juvenilen DM, häufig mit Tumorerkrankungen assoziiert, ein besonders hohes Tumorrisiko haben Patienten mit anti-TIF-1Y-Antikörpern [12]. Das Risiko einer Tumorerkrankung ist im ersten Jahr nach Manifestation der Myositis am größten, ein Tumorscreening sollte bis 3 5 Jahre nach der Diagnose erfolgen. Die häufigsten Malignome umfassen Mammakarzinome und Adenokarzinome der Lunge und des Darms. Neben Befall der Haut und Muskulatur sind bei der DM unabhängig vom Antikörperstatus auch weitere Organmanifestationen (Niere, Lunge, Herz) möglich.

Histologisch zeigt die Dermatomyositis ein charakteristisches Bild mit

- perifaszikulärer Muskelfaseratrophie,

- einer Komplement-vermittelten Mikroangiopathie sowie

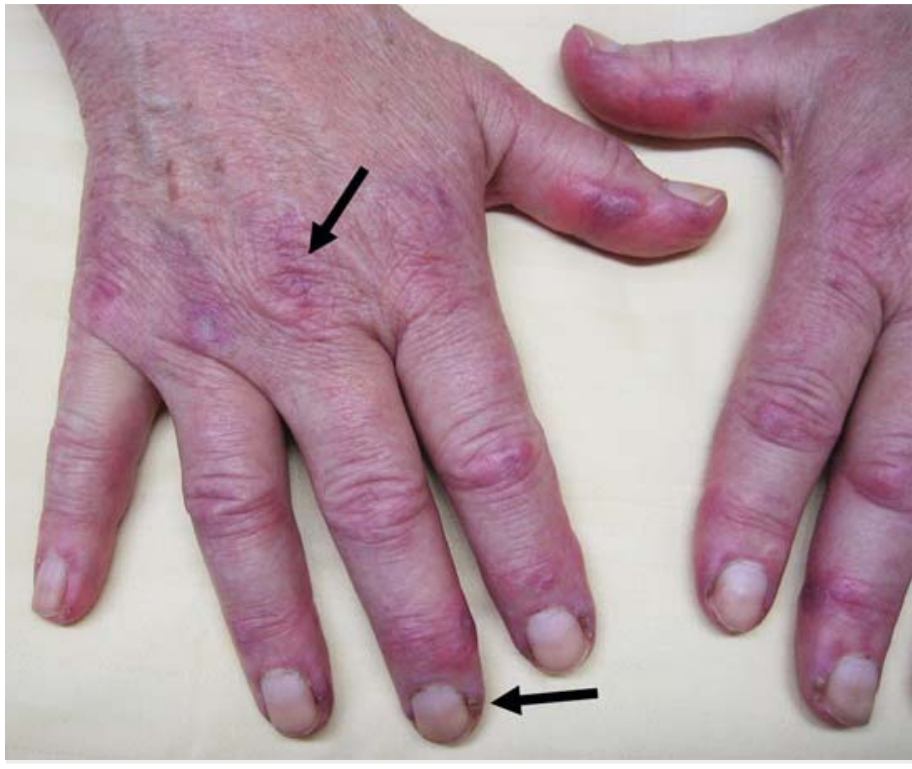

- Abb.1 Erythem und Hyperkeratosen an der Streckseite der Hände sowie periunguale Veränderungen.

- perivasalen entzündlichen Infiltraten im interfaszikulären und perimysialen Bindegewebe [9].

Tab. 1 Idiopathische inflammatorische Myopathien und typische histologische Veränderungen.

\begin{tabular}{|c|c|}
\hline Idiopathische inflammatorische Myopathie & Typische histologische Veränderungen \\
\hline Dermatomyositis & $\begin{array}{l}\mathrm{CD} 4^{+}-\mathrm{T} \text { - und CD20 } 0^{+}-\mathrm{B}-\text { Lymphozyten } \\
\mathrm{CD} 68^{+}-\text {Monozyten/Makrophagen } \\
\text { v. a. perivaskulär ( } \triangleright \text { Abb. } 2 \text { b) } \\
\text { perifaszikuläre Faseratrophie und Aufregulation HLA-I } \\
\text { Ablagerung von C5b-9 und Verlust von endomysialen Kapillaren }\end{array}$ \\
\hline Polymyositis & $\begin{array}{l}\text { entzündliche Infiltrate im Endomysium } \\
\mathrm{CD} 4^{+} \text {- und CD8 } 8^{+} \text {-T-Lymphozyten } \\
\mathrm{CD} 68^{+} \text {-Monozyten/Makrophagen } \\
\text { Invasion vitaler Muskelfasern durch zytotoxische CD8 }{ }^{+}-\text {T-Lymphozyten ( } \triangleright \text { Abb. 2c) } \\
\text { HLA-I-Aufregulation an Muskelfasern ( } \triangleright \text { Abb. } 2 \text { d) }\end{array}$ \\
\hline immun mediierte nekrotisierende Myopathie & $\begin{array}{l}\text { diffuse Fasernekrosen und Regeneratfasern ( } \triangleright \text { Abb. } 2 \text { a) } \\
\text { spärlich entzündliche Infiltrate (CD68 } \\
\text { Aufregulation von HLA-I-Antigen an Muskelfasern } \\
\text { Ablagerung C5b-9 an Kapillaren oder der Oberfläche von Muskelfasern }\end{array}$ \\
\hline Einschlusskörpermyositis & $\begin{array}{l}\mathrm{CD} 4^{+} / \mathrm{CD} 8^{+}-\mathrm{T} \text {-Lymphozyten und } \mathrm{CD} 68^{+}-\text {Monozyten/Makrophagen im Endomysium } \\
\text { Invasion vitaler Muskelfasern durch zytotoxische CD8+-T-Lymphozyten } \\
\text { HLA-I-Aufregulation an Muskelfasern sowie Atrophie } \\
\text { neurogene und degenerative Veränderung von Muskelfasern („rimmed Vacuoles“, \ Abb. 3a; } \\
\text { abnorme Proteinablagerung, \ Abb. } \mathbf{3 b} \text {; COX-negative Fasern) und endomysiale Fibrose }\end{array}$ \\
\hline Overlap-Myositis & $\begin{array}{l}\text { unspezifische entzündliche Infiltrate } \\
\text { HLA-I-Aufregulation an Muskelfasern } \\
\text { Ablagerung C } 5 \text { b- } 9 \text { an der Oberfläche von Muskelfasern }\end{array}$ \\
\hline Anti-Synthetase-Syndrom & $\begin{array}{l}\text { perifaszikuläre Fasernekrosen und Regeneratfasern } \\
\text { Destruktion des perimysialen Bindegewebes }\end{array}$ \\
\hline
\end{tabular}



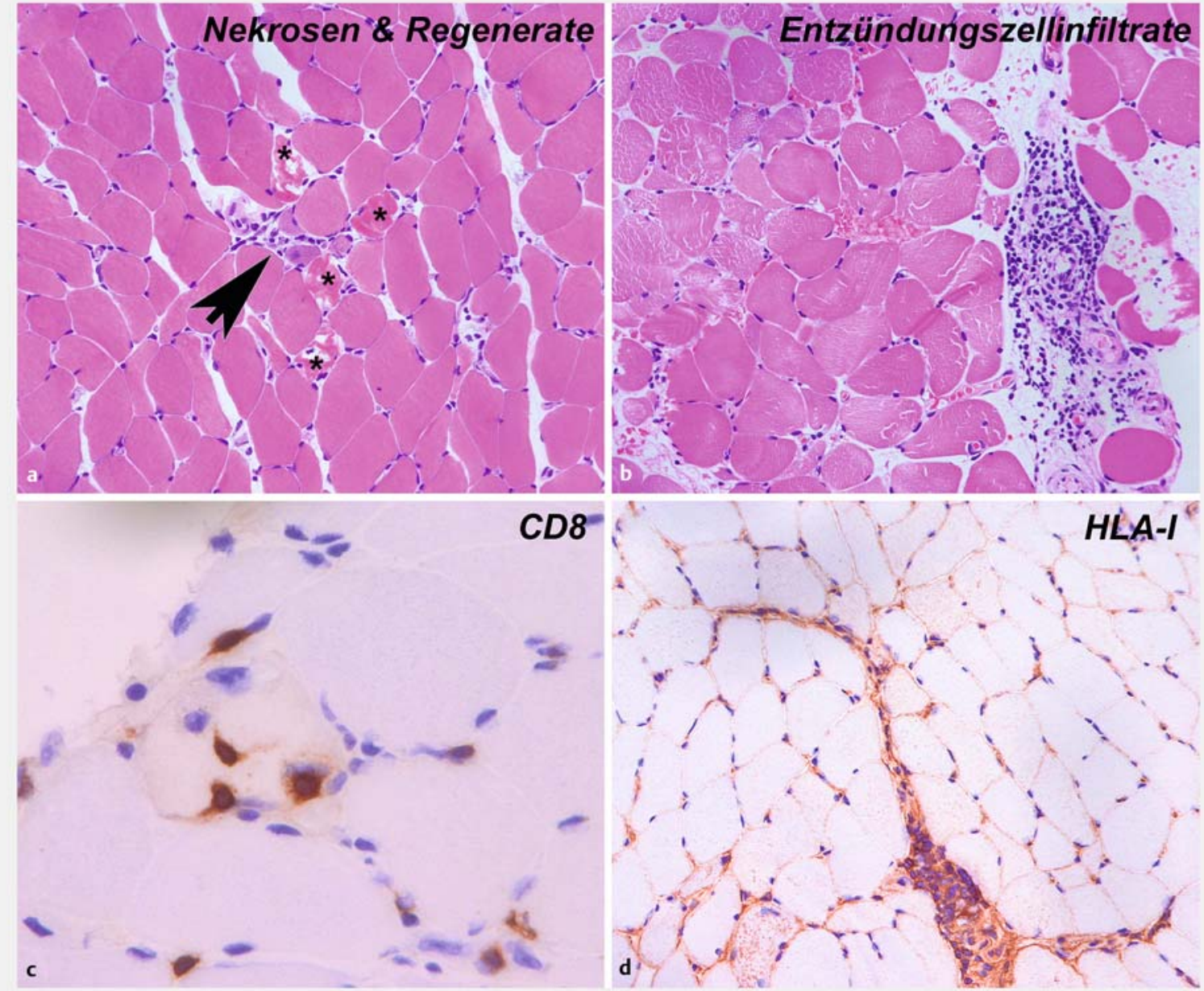

Abb.2 Idiopathische inflammatorische Myopathien und typische histologische Veränderungen (zur Verfügung gestellt von Frau Priv.-Doz. Dr. Ellen Gelpi, Abt. für Neuropathologie der Universitätsklinik für Neurologie, Medizinische Universität Wien, mit freundlicher Genehmigung). a a. Entzündliche Myopathien, insbesondere die immun-mediierte nekrotisierende Myopathie, charakterisieren sich durch Muskelfasernekrosen (Sterne) und Regeneratfasern (Pfeil). b Perivaskuläres entzündliches Infiltrat bei der DM. c Bei der Polymyositis zeigt sich eine Invasion von intakten Muskelfasern durch CD8-positive T-Lymphozyten. $\mathbf{d}$ Es zeigt sich zumeist eine Aufregulierung von HLA-Klasse-I-Antigen.

Idiopathische inflammatorische Myopathien und typische histologische Veränderungen sind in > Tab. 1 sowie $>$ Abb. 2 und $>$ Abb. $\mathbf{3}$ dargestellt.

\section{Merke}

Die Dermatomyositis geht zumeist mit typischen Hautveränderungen einher, selten können diese der Muskelschwäche nachfolgen. In diesem Fall kann die Diagnose zumeist mit einer Muskelbiopsie gesichert werden. Bei der DM des Erwachsenen soll bis 3-5 Jahre nach Erstdiagnose ein Tumorscreening erfolgen.

\section{Merke}

Bei fehlendem Nachweis eines soliden Tumors muss bei der Dermatomyositis, besonders bei Nachweis von Anti-TIF 1Y-Antikörpern, auch an maligne hämatologische Erkrankungen gedacht werden.

\section{Antisynthetase-Syndrom}

Das Antisynthetase-Syndrom wird neuerdings als eigene Entität betrachtet und umfasst einen Symptomkomplex bestehend aus

- Myositis,

- Arthritiden/Arthralgien,

- Fieberschüben,

- Raynaud's Phänomen und

- interstitieller Lungenerkrankung (ILD). 


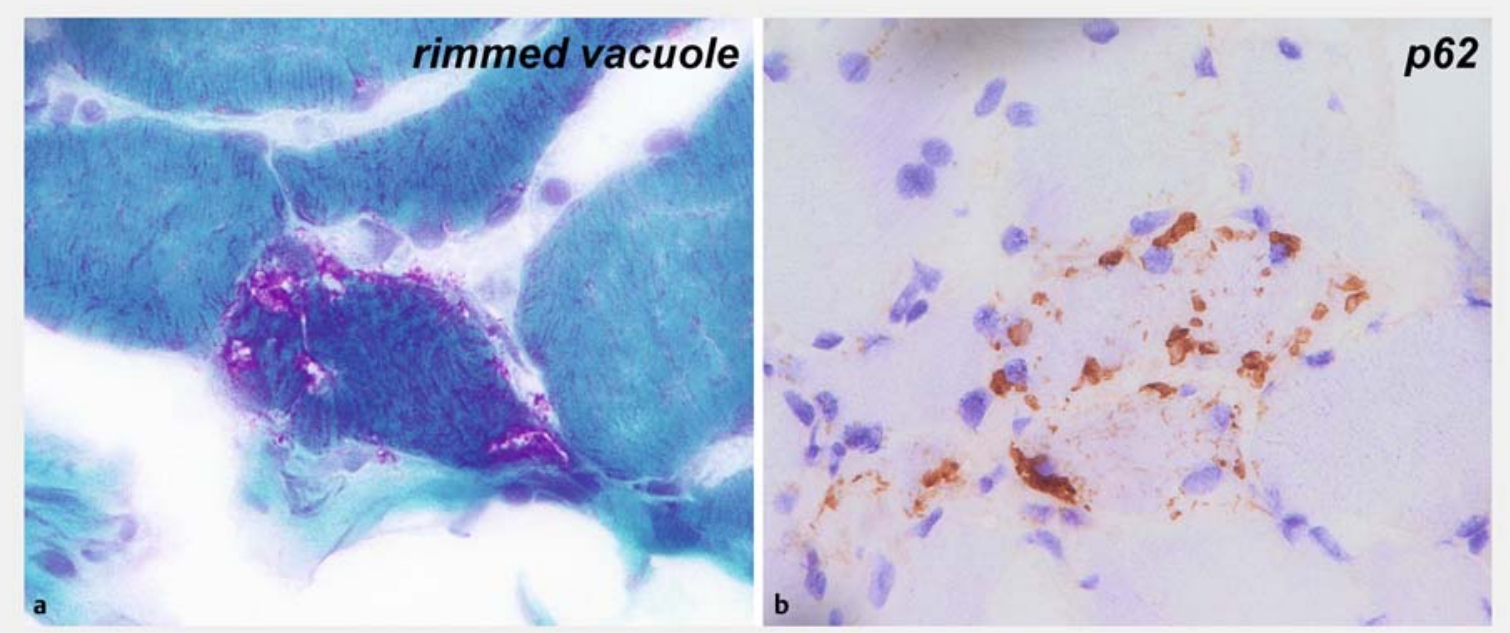

Abb.3 Einschlusskörpermyositis/-myopathie (zur Verfügung gestellt von Frau Priv.-Doz. Dr. Ellen Gelpi, Abt. für Neuropathologie der Universitätsklinik für Neurologie, Medizinische Universität Wien, mit freundlicher Genehmigung). a Bei der Einschlusskörpermyositis/-myopathie zeigen sich zusätzlich sog. geränderte Vakuolen („rimmed Vacuoles“), die gut in der GomoriTrichrom-Färbung zu identifizieren sind. b Diese Vakuolen zeigen deutliche Ablagerungen des Autophagie-assoziierten Proteins p62 (braunes Signal).

\section{FALLBEISPIEL}

\section{Fall 1: Dermatomyositis}

Bei Erstvorstellung 55-jähriger männlicher Patient. Beginn der Symptomatik mit Brennen und Rötung der Kopfhaut, im Gesicht und Brustbereich. Einige Wochen zuvor litt er unter oralen Aphthen. Binnen 2 Wochen nach Beginn der Hauterscheinungen kam es zu einer körperlichen Leistungsminderung sowie Myalgien im Schulter- und Beckengürtelbereich. Die Creatinkinase war auf 5600 U/l erhöht. Im EMG waren myopathisch veränderte Potenziale und Denervierung nachweisbar.

Trotz Therapiebeginn mit Steroiden per os in hoher Dosierung $(80 \mathrm{mg} / \mathrm{Tag})$ und Ebetrexat kam es zu einer klinischen Verschlechterung mit progredienten proximalen Paresen der oberen Extremitäten und der Stammmuskulatur, schwerer Dysphagie und UIzerationen der Fingerkuppen ( $\triangleright$ Abb.4).

Unter Gabe intravenöser Immunglobuline konnte eine Stabilisierung der Paresen erreicht werden. Aufgrund der Schluckstörung musste jedoch vorübergehend eine perkutane endoskopische Gastrostomie (PEG-Sonde) zur Ernährung angelegt werden. Im Serum konnten Anti-TIF 1y-Antikörper nachgewiesen werden. In einem ausgedehnten Tumor- screening mittels Ganzkörper-CT, Gastroskopie, Koloskopie und FDG-PET bestand kein Hinweis für einen soliden Tumor. Eine Splenomegalie und eine vorbekannte Thrombozytose (maximal $732 \mathrm{G} / \mathrm{I}$ ) gaben Anlass zu einer hämatologischen Abklärung. Hier wurde die Diagnose eines myelodysplastischen Syndroms mit JAK2 (V617F)-Mutation gestellt. Im Verlauf musste die Therapie mit Ruxolitinib, Mycophenolat-Mofetil und Rituximab erweitert werden, darunter kam es zu einer Normalisierung der Muskelkraft. Die Hautveränderungen sprachen weniger gut auf die Therapie an.

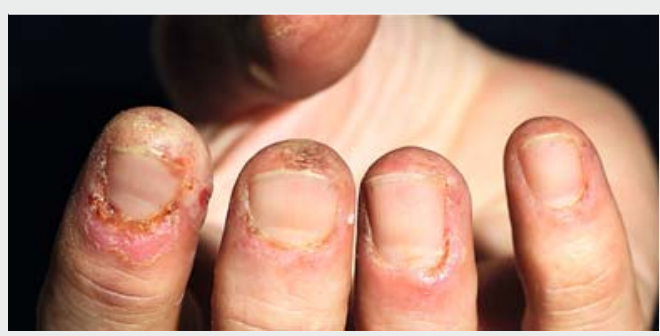

Abb. 4 Ulzera an den Fingerakren bei einem Patienten mit anti-TIF 1Y-Antikörpern als Hinweis für eine schwere entzündliche Vaskulopathie. 
Die ILD kann schwer ausgeprägt sein und bestimmt die Prognose der Erkrankung. Die Haut der Hände weist eine charakteristische Verhornungsstörung und Rhagaden an den Fingerkuppen auf („Mechanic's Hands“) [14]. Tumorerkrankungen treten seltener als bei der Dermatomyositis auf.

Eine Überschneidung mit einer rheumatoiden Arthritis mit anti-CCP-Antikörpern ist möglich. Die wichtigsten Antisynthetase-Autoantikörper (ASAs) sind anti-jo-1, und anti-(Aminoacyl-tRNA-Synthetasen) PL7, PL12, EJ, OJ, KS, Zo, Ha [11]. In Muskelbiopsien wurden als relativ spezifischer Befund perifaszikuläre Muskelfasernekrosen und eine Destruktion des perimysialen Bindegewebes beschrieben [15].

Dermatomyositis mit Antikörpern gegen Melanoma-Differentiation-Associated Gene 5 (anti-MDA5)

Die anti-MDA5 ist durch ausgeprägte (teils ulzerierende) Hautveränderungen, Polyarthralgien und interstitielle Lungenerkrankung (ILD) charakterisiert, wohingegen klinische Zeichen einer Myositis mild sein oder fehlen können („amyopathische DM“). Besonders im asiatischen Raum wurde eine hohe Mortalitätsrate durch eine rasch progrediente ILD beschrieben [16].

An einer großen französischen Kohorte von 121 Patienten wurden kürzlich 3 klinische Phänotypen mit unterschiedlicher Prognose identifiziert:

- rasch progrediente ILD mit sehr hoher Mortalität,

- dermato-rheumatologische Symptome (Rash, Arthralgien) mit guter Prognose sowie

- schwere kutane Vaskulopathie (Hautulzerationen) und proximale Muskelschwäche mit intermediärer Prognose [17].

Merke

Eine sorgfältige klinische Untersuchung muskulärer und extramuskulärer Manifestationen und Bestimmung Myositis-spezifischer Antikörper ermöglicht häufig die Untergliederung in homogene Unterformen der Myositiden mit unterschiedlicher Prognose und Tumorrisiko.

\section{Myositiden ohne Hautmanifestationen mit symmetrischer proximaler Schwäche}

\section{Polymyositis (PM)}

Die Polymyositis betrifft überwiegend das junge bzw. mittlere Erwachsenenalter. Die Paresen können sich schleichender als bei der Dermatomyositis entwickeln. Hauterscheinungen fehlen, sodass der Beginn der Erkrankung oft schwieriger zu bestimmen ist. Das Verteilungsmuster der Muskelschwäche entspricht überwiegend dem der DM, kann aber auch distale Muskeln be- treffen. Die Creatinkinase-Werte können sehr hoch sein (bis zu 50-fach über dem Normwert). Eine Assoziation mit Tumoren (Adenokarzinome) ist seltener als bei der Dermatomyositis [18]. Myositis-spezifische Antikörper sind bei der PM bisher nicht nachgewiesen worden [19]. Eine PM kann isoliert oder als Teilmanifestation einer anderen rheumatologischen Erkrankung wie Lupus erythematodes oder Sklerodermie im Sinne eines Overlap- Syndroms auftreten [9].

Die klinische Abgrenzung einer isolierten PM zu metabolischen Myopathien oder Muskeldystrophien kann unter Umständen Schwierigkeiten bereiten. In einem frühen Krankheitsstadium kann eine Einschlusskörpermyositis als PM fehldiagnostiziert werden.

Die Muskelbiopsie ist durch entzündliche Infiltrate im Endomysium, Aufregulation von HLA-I-Antigen am Sarkolemm zahlreicher Muskelfasern und Invasion nichtnekrotischer Muskelfasern durch zytotoxische CD8 ${ }^{+}-\mathrm{T}_{-}$ Zellen charakterisiert.

\section{Immun mediierte nekrotisierende Myopathie (IMNM)}

Die IMNM wurde in den letzten Jahren als eigener Subtyp charakterisiert [20]. Das Verteilungsmuster der Paresen betrifft wie bei der Dermatomyositis und der Polymyositis die proximale und axiale Muskulatur, wobei die Muskelschwäche sehr ausgeprägt sein kann. Myalgien treten bei bis zu 80 \% der Patienten auf, eine Dysphagie ist häufig. Muskelenzyme können exzessiv erhöht sein (CK>50-fach, LDH, GOT, GPT). Es besteht eine starke Assoziation mit Antikörpern gegen SRP (Signal Recognition Particle), einem Protein des endoplasmatischen Retikulums, oder gegen HMGCR (Hydroxy-3-Methylglutaryl Coenzym A Reduktase) [21]. Extramuskuläre Manifestationen sind selten oder mild.

Die immun mediierte nekrotisierende Myopathie mit SRP-Antikörpern tritt bevorzugt im mittleren Lebensalter auf ( $\triangleright$ Abb.5) und zeigt einen schwereren Verlauf als die IMNM mit HMGCR-Antikörpern. Letztere betrifft eher das höhere Lebensalter und hat eine hohe Assoziation mit einer Statin-Exposition und ein höheres Tumorrisiko [22].

Im Gegensatz zur Statin-induzierten Myopathie (toxisch-metabolische Schädigung der Muskelfasern) zeigt die immun mediierte nekrotisierende Myopathie durch das Absetzten der Statine keine Besserung, sondern erfordert eine konsequente immunsuppressive Therapie. Wenn aufgrund kardiovaskulärer Komorbidität eine Lipidsenkung erforderlich ist, kann eine Therapie mit einem PCSK9-Inhibitor erwogen werden. Selten kann eine IMNM mit HMGCR-Antikörpern auch bei jüngeren Patienten ohne Statin-Exposition auftreten. 


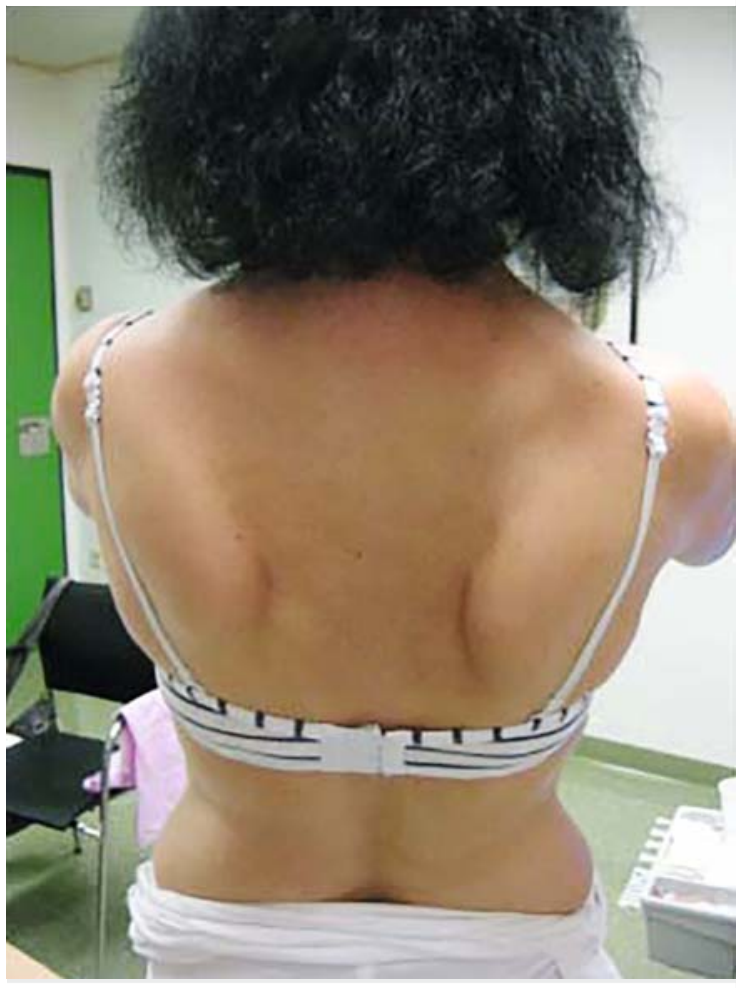

- Abb.5 Atrophie der Schultergürtelmuskulatur und Scapula alata bei therapierefraktärer IMNM (immun mediierte nekrotisierende Myopathie) mit SRP-Antikörpern ( $\mathrm{SRP}=$ Signal Recognition Particle).

Die Muskelbiopsie zeigt bei beiden Formen Muskelfasernekrosen und Regeneratfasern, jedoch nur minimale bis fehlende lymphozytäre Entzündungszellinfiltrate. Typischerweise vorhanden sind

\section{FALLBEISPIEL}

\section{Fall 2: Immun mediierte nekrotisierende Myopathie mit SRP-Antikörpern}

Es handelt sich um eine bei Krankheitsmanifestation 58-jährige Patientin. Initial traten Muskelschmerzen über mehrere Monate und eine subakute proximale Schwäche der unteren Extremitäten auf. Die Creatinkinase bei Aufnahme lag bei 10,786U/I. Klinisch zeigte sich eine proximale Tetraparese. An der Haut waren keine Auffälligkeiten zu beobachten. Im EMG waren myogene Schädigungszeichen mit Denervierung nachzuweisen. Die Antikörpertestung ergab einen positiven Befund für anti-SRP-Antikörper. Die Muskelbiopsie ergab eine nekrotisierende Myopathie mit spärlichen entzündlichen Infiltraten. Das Tumorscreening war negativ.

Initial zeigte die Patientin ein gutes Ansprechen auf eine intravenöse Hochdosis-Steroidtherapie und an-
- Eine Aufregulation des Major-Histokompatibilitätskomplexes,

- meist spärliche Infiltration durch Makrophagen und

- Ablagerung des terminalen Komplementkomplexes an der Muskelfasermembran nicht-nekrotischer Muskelfasern.

Diese Befunde untermauern die Immunpathogenese der Erkrankung [23].

\section{Merke}

Bei Patienten mit ausgeprägten Paresen und exzessiver Creatinkinase-Erhöhung muss an eine immun mediierte nekrotisierende Myopathie (IMNM) gedacht werden. Bei HMGCR-Antikörpern geht IMNM zumeist eine Statin-Exposition voraus.

\section{Myositis ohne Hautmanifestationen mit asymmetrischer proximaler und distaler Schwäche}

\section{Einschlusskörpermyositis (IBM)}

Die Einschlusskörpermyositis ist die häufigste erworbene Myopathie des höheren Lebensalters und zeigt ein typisches Verteilungsmuster mit Paresen und Atrophien der langen Fingerbeuger und Knieextensoren, die zu einer progredienten Feinmotorik- und Gangstörung führen. Die Ausprägung ist asymmetrisch, an den oberen Extremitäten ist die nicht-dominante Seite häufig stärker betroffen. Armabduktion oder Hüftbeugung sind im Gegensatz zu den anderen Myositiden nur gering oder erst spät im Krankheitsverlauf betroffen. Eine Dysphagie ist häufig und kann auch das initiale Symptom sein. schließende orale Steroideinnahme. Auf Gabe von Azathioprin kam es zu einer massiven yGT-Erhöhung, sodass die Indikation für Rituximab gestellt wurde. Entgegen Daten aus der Literatur hatte die Patientin keinen Benefit durch die Gabe von $2 \times 1000$ mg Rituximab im Abstand von 2 Wochen und entschied sich gegen eine neuerliche Medikation. Unter intravenösen Immunglobulinen und Mycophenolat-Mofetil kam es zu einer Stabilisierung der Paresen, orale Steroide wurden aufgrund von Hautblutungen abgesetzt.

Nach 3 Jahren Krankheitsdauer hat die Patientin geringe Restparesen axial und für die Hüftbeugung, beklagt jedoch eine verminderte Ausdauer. Die aktuelle Creatinkinase ist mit $359 \mathrm{U} / \mathrm{I}$ noch gering erhöht. 
FALLBEISPIEL

Fall 3: Immun mediierte nekrotisierende Myopathie mit HMGCR-Antikörpern

Eine bis dato sehr sportliche Patientin bemerkte im Alter von 63 Jahren einen Leistungseinbruch und eine proximale Schwäche der Beine, die sie zunächst besonders beim Skifahren beeinträchtigte. Aufgrund einer Hypercholesterinämie und eines Plaques in der rechten Karotis nahm sie seit 3 Jahren Atorvastatin $40 \mathrm{mg}$ ein.

Bei einer Blutabnahme fand sich eine CreatinkinaseErhöhung auf $10000 \mathrm{U} / \mathrm{l}$. Atorvastatin wurde daraufhin abgesetzt, die CK-Erhöhung nahm über die nächsten 4 Wochen jedoch nicht ab, die Muskelschwäche steigerte sich noch.

Bei stationärer Aufnahme bot die Patientin proximale Paresen vom Kraftgrad 4 an Armen und Beinen und milde Myalgien. Im EMG waren deutliche Zeichen einer myogenen Schädigung mit pathologischer Spontanaktivität fassbar. In der Muskelbiopsie fanden sich Muskelfasernekrosen und Regeneratfasern, spärlich Makrophagen, eine Aufregulation von HLA-I und geringe Komplementablagerungen an der
Oberfläche von Muskelfasern. Im Serum konnten HMGCR-Antikörper nachgewiesen werden. An Laborbefunden fiel weiters ein positiver Elispot bei bekannter Tuberkulose ihrer Schwester in der Jugend auf. Eine Lymphknotenbiopsie zeigte keine spezifischen Veränderungen.

Auf Gabe von Kortison $60 \mathrm{mg} / \mathrm{Tag}$ kam es in den folgenden 2-3 Wochen zu einer deutlichen Besserung der Paresen und Rückgang der HyperCKämie. In der Folge wurde Kortison reduziert und Methotrexat als steroidsparende Substanz verordnet. Aufgrund unzureichender Symptomkontrolle wurde eine Therapie mit intravenösen Immunglobulinen begonnen. Methotrexat wurde aufgrund rezidivierender Harnwegsinfekte und gastrointestinaler Beschwerden wieder abgesetzt.

Unter regelmäßiger IVIG-Gabe alle 6 Wochen und niedrig dosierter Steroideinnahme besteht eine minimale Schwäche für die Armabduktion bei guter körperlicher Belastbarkeit. Die CK-Werte sind mit 2000 U/I jedoch weiterhin erhöht.

\section{FALLBEISPIEL}

\section{Fall 4: Einschlusskörpermyositis (IBM)}

Es handelt sich um einen 63-jährigen Patienten, der sich in der neuromuskulären Ambulanz vorstellt. Eine HyperCKämie um $1000 \mathrm{U} / \mathrm{l}$ ist bereits vor 14 Jahren im Rahmen einer Routineuntersuchung aufgefallen. Bei einer neurologischen Untersuchung vor 10 Jahren waren keine Paresen oder Atrophien nachzuweisen. Das EMG war minimal unspezifisch verändert, es fanden sich einzelne kurze komplexrepetitive Entladungen.

Von einer weiteren Abklärung wurde vorerst abgesehen. 1 Jahr vor Wiedervorstellung bemerkte der Patient eine progrediente Schwäche und Atrophie des M. quadriceps links > rechts. Es bestanden Paresen für die Fingerendgliedbeugung.

Die Creatinkinase lag bei Wiedervorstellung bei 527 U/I. In der Neurografie zeigte sich eine distal-symmetrische sensomotorische Neuropathie der unteren Extremitäten mit axonalen und demyelinisierenden Schädigungszeichen. Im EMG fanden sich scharf positive Wellen, Fibrillationspotenziale und komplex-repetitive Entladungen, die Muskelaktionspotenziale zeigten häufig eine verkürzte Potenzialdauer, die Amplituden waren teils erniedrigt und polyphasisch, gelegentlich auch überhöht und schlank konfiguriert. Anti-cN1a-Antikörper waren positiv. Die Muskelbiopsie des M. vastus lateralis links ergab entzündliche Infiltrate im Endomysium und chronisch degenerative Veränderungen der Muskelfasern mit geränderten Vakuolen - typisch für eine IBM. 3 Serien mit intravenösen Immunglobulinen ergaben keinen Benefit. Durch eine Physiotherapie konnte eine leichte Stabilisierung der Gangunsicherheit erreicht werden.

Als Komorbiditäten sind ein Insulin-pflichtiger Diabetes mellitus Typ 2 und eine kombinierte Hyperlipidämie bekannt. Aufgrund einer Kontraindikation gegenüber Statinen erfolgt eine Lipid-senkende Therapie mit Repatha (Evolocumab) in 14-tägigen Abständen. 
Das Manifestationsalter liegt zumeist nach dem 50. Lebensjahr, der Verlauf ist langsam progredient, sodass die Diagnose oft verzögert gestellt wird (durchschnittliche Latenz ca. 6 Jahre). Männer sind doppelt so häufig betroffen wie Frauen.

Die Creatinkinase-Werte sind leicht bis mäßig erhöht mit Werten unter 1000 U/I. Bei 30-60\% der PatientInnen sind Antikörper gegen cytosolische 5'-Nucleotidase nachweisbar (anti-cN1a-Antikörper, Synonym: antiMup-44-Antikörper).

Merke

Die Diagnosesicherung einer Einschlusskörpermyositis erfordert eine Muskelbiopsie.

Typischerweise zeigen sich entzündliche Infiltrate im Endomysium, die sich überwiegend aus hochdifferenzierten CD8 ${ }^{+}$-T-Lymphozyten und CD68-positiven Monozyten zusammensetzen, aber auch plasmozytoide Zellen der B-Zellreihe können nachweisbar sein. Ähnlich wie bei der Polymyositis besteht eine weitläufige Aufregulation von HLA-I-Antigen am Sarkolemm und gelegentlich eine Faserinvasion durch zytotoxische CD8 ${ }^{+}$-T-Zellen.

Charakteristisch für die IBM ist die Koexistenz chronisch degenerativer Veränderungen mit felderförmigen Faseratrophien und Fibrosierung des endomysialen Bindegewebes. Im Sarkoplasma degenerierender Muskelfasern können geränderte Vakuolen und abnorme Ablagerungen von verschiedenen Proteinen wie $\beta$ Amyloid, phosphoryliertem Tau-Protein, Ubiquitin, p62 und TDP43 etc. vorliegen, diese können immunhistochemisch detektiert werden. Auf ultrastruktureller Ebene sind tubulo-filamentöse Einschlüsse von 15-18 $\mathrm{nm}$ typisch [24].

Merke

Die Einschlusskörpermyositis (IBM) tritt zumeist jenseits des 50. Lebensjahres auf und zeigt einen schleichend progredienten Verlauf. Paresen und Atrophien betreffen besonders die Kniestrecker und langen Fingerbeuger.

\section{Diagnostik}

\section{Nadelelektromyografie (EMG)}

Mittels EMG können in den betroffenen Muskeln Veränderungen einer myogenen Schädigung nachgewiesen werden. Im akuten Stadium können scharf positive Wellen und Fibrillationen fassbar sein, die Amplituden der Muskelaktionspotenziale (MUAPs) sind erniedrigt und polyphasisch aufgesplittert, das Interferenzmuster zeigt eine abnorm frühe Rekrutierung motorischer Einheiten. Im chronischen Stadium können die Amplitu- den der MUAPs wieder zunehmen, die Dauer der Potenziale bleibt jedoch verkürzt. So können besonders bei der Einschlusskörpermyositis niedrige und überhöhte, jedoch „schlanke“ Potenziale nebeneinander bestehen, eventuell können komplex-repetitive Entladungen abgeleitet werden.

Eine Neurografie bzw. repetitive Stimulation kann bei entsprechender Anamnese zum Ausschluss anderer neuromuskulärer Erkrankungen sinnvoll sein (z. B. Guillain-Barré-Syndrom, chronisch inflammatorische demyelinisierende Neuropathie, Myasthenia gravis).

\section{Bildgebung}

MRT

Mittels MRT können entzündliche Veränderungen der Muskulatur in Fett-unterdrückten Sequenzen (ShortTau Inversion Recovery; STIR) als Ödem dargestellt werden. In T1-gewichteten Sequenzen können das Ausmaß der Muskelatrophie und Fettumwandlung beurteilt werden. Das Verteilungsmuster der Veränderungen kann in der Abgrenzung der verschiedenen Myositiden hilfreich sein, so zeigt die Dermatomyositis im Gegensatz zu Polymyositis und immun mediierter nekrotisierende Myopathie ein prominentes Ödem der Faszien [25].

\section{Sonografie}

Anhand rezenter Studien gewinnt auch die hochauflösende Sonografie eine zunehmende Bedeutung für die Beurteilung von Muskelmasse und Struktur, die auch eine dynamische Untersuchungen bei Bewegung ermöglicht [26]. Quantitative Untersuchungen der Echogenität und Dicke betroffener Muskeln können zur Differenzierung zwischen Polymyositis/Dermatomyositis und Einschlusskörpermyositis beitragen [27].

Die Shear-Wave-Elastografie erscheint besonders geeignet, um einen Verlust an Elastizität und Fibrosierung im Verlauf einer Myositis darzustellen [26].

\section{Antikörperdiagnostik}

Bei $>60 \%$ bis $70 \%$ der Patienten mit Myositis können Myositis-spezifische bzw. Myositis-assoziierte Antikörper (MSA, MAA) nachgewiesen werden. Durch die Assoziation mit speziellen Krankheitsmerkmalen unterstützen sie nicht nur die Immunpathogenese der Erkrankung, sondern ermöglichen auch die Subtypisierung im homogene Krankheitsgruppen mit prognostischer und therapeutischer Relevanz [11].

Eine Paneldiagnostik der wichtigsten Antikörper wird kommerziell angeboten und steht für die Routinediagnostik zur Verfügung. Die Bestimmung von MSA bei unspezifischen Beschwerden wie Myalgien und Fatigue ohne Paresen oder Creatinkinase-Erhöhung ist nicht 
zielführend, da in dieser Situation die Relevanz positiver Befunde unklar ist.

\section{Biopsie}

Bei eindeutiger klinischer Konstellation (rascher Krankheitsbeginn, typische Hautveränderungen, über Wochen persistierende Creatinkinase-Erhöhung, symmetrische proximale Paresen und Zeichen einer Myopathie im EMG) wird auf eine Muskelbiopsie häufig verzichtet. Bei schweren bzw. therapierefraktären Fällen ist jedoch eine möglichst frühzeitige Diagnosesicherung durch eine Biopsie sinnvoll.

\section{Durchführung}

Eine bereits begonnene Therapie mit Kortison sollte mindestens 3 Wochen vor der Biopsie pausiert werden (falls klinisch vertretbar), da sich durch die Einnahme von Kortison entzündliche Infiltrate zurückbilden können.

Der für die Biopsie ausgewählte Muskel soll klinisch mäßig betroffen sein (Faustregel>Parese/KG 4) und darf keine fortgeschrittene Atrophie aufweisen („Endstage Disease“), da in diesem Fall der zugrundeliegende Prozess durch Fibrose und Umwandlung in Fettgewebe eventuell nicht mehr zugeordnet werden kann.

Essenziell ist die Aufarbeitung in einem für Muskelbiopsien spezialisierten Labor. Zahlreiche enzym- und immunhistochemische Färbungen sind nur auf Gefriermaterial möglich.

FAZIT

Take Home Message

Die Diagnose einer Myositis basiert auf der Anamnese, klinischen Untersuchung und Ergebnis der Zusatzuntersuchungen. Das EMG und MRT können eine Muskelschädigung untermauern, sind aber per se nicht spezifisch. Für die sichere Diagnose einer Polymyositis oder Einschlusskörpermyositis wird weiterhin eine Muskelbiopsie benötigt.

\section{IIM-Therapieprinzipien}

Im akuten Schub ist die Therapie mit Glukokortikoiden aufgrund des raschen Wirkungseintritts unverändert 1 . Wahl. Je nach Schweregrad der Myositis wird eine Startdosis Prednisolon 0,5-1 mg/kgKG empfohlen. Bei schwerer Krankheitsmanifestation kann eine intravenöse Pulstherapie mit jeweils 1000 mg über 3 Tage verabreicht werden. Wichtig ist, dass eine ausreichend hohe Dosis bis zur klinischen Stabilisation über mehrere Wochen aufrechterhalten wird und die Dosisreduktion langsam schrittweise erfolgt. Als steroidsparende Substanzen stehen Azathioprin und Methotrexat zur Verfügung.

Bei schwerer Organmanifestation wie interstitielle Lungenerkrankung (ILD) können Ciclosporin A und Mycophenolat-Mofetil zum Einsatz kommen. Auch Kombinationen sind bei therapierefraktärer Myositis möglich.

Die Wirkung intravenöser Immunglobuline wurde bei Dermatomyositis nachgewiesen und lässt sich mit allen oben genannten Substanzen kombinieren. Rituximab führt zur Depletion von CD20+-B-Zellen und kann besonders bei Vorliegen Myositis-spezifischer Antikörper die Krankheitsaktivität verringern und helfen, Steroide einzusparen. Für die Einschlusskörpermyositis konnte bisher kein Benefit immunsuppressiver bzw. immunmodulierender Medikament gezeigt werden.

Eine physikalische Therapie und moderates Muskeltraining ist bei allen IIM und Einschlusskörpermyositiden wesentlicher Bestandteil der Behandlung. Als weiterführende Literatur s. [19, 28]. 


\section{KERNAUSSAGEN}

- Idiopathische inflammatorische Myopathien (Myositiden) sind heterogene Erkrankungen, die neben dem gemeinsamen Nenner einer proximalen/axialen Muskelschwäche und HyperCKämie unterschiedliche extramuskuläre Organmanifestationen und ein Tumorrisiko aufweisen können.

- Die Bestimmung Myositis-spezifischer Antikörper kann bei entsprechender Klinik zur Unterteilung in homogene Subtypen beitragen.

- Die Einschlusskörpermyositis (IBM) unterscheidet sich von anderen Myositiden durch ein häufig asymmetrisches Verteilungsmuster aus distalen Paresen der oberen und proximalen Paresen der unteren Extremitäten sowie die Koexistenz entzündlicher und chronischer degenerativer Veränderungen in der Muskelbiopsie. Bisher verfügbare immunmodulatorische und immunsuppressive
Substanzen können die Progredienz der IBM nicht aufhalten.

- Die Diagnose einer Myositis basiert auf der Anamnese, klinischen Untersuchung und Ergebnis der Zusatzuntersuchungen. Das EMG und MRT können eine Muskelschädigung untermauern sind aber per se nicht spezifisch. Für die sichere Diagnose einer Polymyositis (PM) oder Einschlusskörpermyositis (IBM) wird weiterhin eine Muskelbiopsie benötigt.

- Auch hereditäre Muskelerkrankungen wie z. B. die fazio-skapulo-humerale Muskeldystrophie, Dystrophinopathien oder Dysferlinopathien können in der Muskelbiopsie unspezifische entzündliche Infiltrate aufweisen, daher ist die genaue Erhebung der Anamnese und der klinischen Präsentation für die richtige Diagnose essenziell.

\section{Interessenkonflikt}

\section{Erklärung zu finanziellen Interessen}

Forschungsförderung erhalten: nein; Honorar/geldwerten Vorteil für Referententätigkeit erhalten: nein; Bezahlter Berater/interner Schulungsreferent/Gehaltsempfänger: nein; Patent/Geschäftsanteile/Aktien (Autor/Partner, Ehepartner, Kinder) an Firma (Nicht-Sponsor der Veranstaltung): nein; Patent/Geschäftsanteile/Aktien (Autor/Partner, Ehepartner, Kinder) an Firma (Sponsor der Veranstaltung): nein.

Erklärung zu nichtfinanziellen Interessen

Österreichische Gesellschaft für Neurologie

\section{Autorinnen/Autoren}

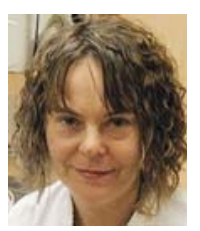

\section{Julia Wanschitz}

Priv.-Doz. Dr. med. Studium der Medizin an der Universität Wien, Ausbildung für Neuropathologie und Neurologie an den Medizinischen Universitäten Wien und Innsbruck. Seit Nov. 2004 Fachärztin für Neurologie. Von Sept. 2008 bis Dez. 2009 Sabbatical am Institute de Myologie, Paris. Habilitation für Neurologie im Sept. 2011. Aktuelle Position stellvertretende Leiterin der neuromuskulären Ambulanz der Universitätsklink für Neurologie, Innsbruck.

\section{Korrespondenzadresse}

\section{OÄ Priv.-Doz. Dr. Julia Wanschitz}

Medizinische Universität Innsbruck

Abteilung für Neurologie

Anichstraße 35

A-6020 Innsbruck

Österreich

julia.wanschitz@i-med.ac.at

Wissenschaftlich verantwortlich gemäß Zertifizierungsbestimmungen

Wissenschaftlich verantwortlich gemäß Zertifizierungsbestimmungen für diesen Beitrag ist Priv.-Doz. Dr. med. Julia Wanschitz, Innsbruck.

\section{Literatur}

[1] Feldman BM, Rider LG, Reed AM et al. Juvenile dermatomyositis and other idiopathic inflammatory myopathies of childhood. Lancet 2008; 371: 2201-2212 doi:10.1016/ S0140-6736(08)60955-1

[2] Mall S, Buchholz U, Tibussek D et al. A large outbreak of influenza B-associated benign acute childhood myositis in Germany, 2007/2008. Pediatr Infect Dis J 2011; 30: e142146 doi:10.1097/INF.0b013e318217e356

[3] Beydon M, Chevalier K, Al Tabaa O et al. Myositis as a manifestation of SARS-CoV-2. Ann Rheum Dis 2020: doi:10.1136/annrheumdis-2020-217573

[4] Megremis S, Walker TDJ, He X et al. Antibodies against immunogenic epitopes with high sequence identity to SARSCoV-2 in patients with autoimmune dermatomyositis. Ann Rheum Dis 2020: doi:10.1136/annrheumdis-2020-217522

[5] Miller FW, Lamb JA, Schmidt J et al. Risk factors and disease mechanisms in myositis. Nat Rev Rheumatol 2018; 14: 255 268 doi:10.1038/nrrheum.2018.48 
[6] Rothwell S, Chinoy H, Lamb JA et al. Focused HLA analysis in Caucasians with myositis identifies significant associations with autoantibody subgroups. Ann Rheum Dis 2019; 78 : 996-1002 doi:10.1136/annrheumdis-2019-215046

[7] Benveniste O, Drouot L, Jouen F et al. Correlation of antisignal recognition particle autoantibody levels with creatine kinase activity in patients with necrotizing myopathy. Arthritis Rheum 2011; 63: 1961-1971 doi:10.1002/art.30344

[8] Khoo T, Stokes MB, Teo K et al. Cardiac involvement in idiopathic inflammatory myopathies detected by cardiac magnetic resonance imaging. Clin Rheumatol 2019; 38: 34713476 doi:10.1007/s10067-019-04678-z

[9] Selva-O'Callaghan A, Pinal-Fernandez I, Trallero-Araguas E et al. Classification and management of adult inflammatory myopathies. Lancet Neurol 2018; 17: 816-828 doi:10.1016/S1474-4422(18)30254-0

[10] Cabral BMI, Edding SN, Portocarrero JP et al. Rhabdomyolysis. Dis Mon 2020; 66: 101015 doi:10.1016/j.disamonth.2020.101015

[11] Betteridge Z, Tansley S, Shaddick G et al. Frequency, mutual exclusivity and clinical associations of myositis autoantibodies in a combined European cohort of idiopathic inflammatory myopathy patients. J Autoimmun 2019; 101: 48-55 doi:10.1016/j.jaut.2019.04.001

[12] Dani L, Holmqvist M, Martinez MA et al. Anti-transcriptional intermediary factor 1 gamma antibodies in cancer-associated myositis: a longitudinal study. Clin Exp Rheumatol 2020; 38: 67-73

[13] Albayda J, Pinal-Fernandez I, Huang W et al. Antinuclear matrix protein 2 autoantibodies and edema, muscle disease, and malignancy risk in dermatomyositis patients. Arthritis Care Res (Hoboken) 2017; 69: 1771-1776 doi:10.1002/acr.23188

[14] Marco JL, Collins BF. Clinical manifestations and treatment of antisynthetase syndrome. Best Pract Res Clin Rheumatol 2020: doi:10.1016/j.berh.2020.101503

[15] Mescam-Mancini L, Allenbach Y, Hervier B et al. Anti-Jo-1 antibody-positive patients show a characteristic necrotizing perifascicular myositis. Brain 2015; 138: 2485-2492 doi:10.1093/brain/awv192

[16] Takada T, Ohashi K, Hayashi M et al. Role of IL-15 in interstitial lung diseases in amyopathic dermatomyositis with anti-MDA-5 antibody. Respir Med 2018; 141: 7-13 doi:10.1016/j.rmed.2018.06.012

[17] Allenbach $Y$, Uzunhan $Y$, Toquet $S$ et al. Different phenotypes in dermatomyositis associated with anti-MDA5 antibody: Study of 121 cases. Neurology 2020: doi:10.1212/ WNL.0000000000009727

[18] Hill CL, Zhang Y, Sigurgeirsson B et al. Frequency of specific cancer types in dermatomyositis and polymyositis: a population-based study. Lancet 2001; 357: 96-100 doi:10.1016/ S0140-6736(00)03540-6
[19] Baig S, Paik J]. Inflammatory muscle disease - An update. Best Pract Res Clin Rheumatol 2020; 34: 101484 doi:10.1016/j.berh.2019.101484

[20] Christopher-Stine L, Casciola-Rosen LA, Hong G et al. A novel autoantibody recognizing $200-k d$ and $100-k d$ proteins is associated with an immune-mediated necrotizing myopathy. Arthritis Rheum 2010; 62: 2757-2766 doi:10.1002/ art. 27572

[21] Watanabe Y, Uruha A, Suzuki S et al. Clinical features and prognosis in anti-SRP and anti-HMGCR necrotising myopathy. J Neurol Neurosurg Psychiatry 2016; 87: 1038-1044 doi:10.1136/jnnp-2016-313166

[22] Allenbach Y, Keraen J, Bouvier AM et al. High risk of cancer in autoimmune necrotizing myopathies: usefulness of myositis specific antibody. Brain 2016; 139: 2131-2135 doi:10.1093/brain/aww054

[23] Mohassel P, Mammen AL. Anti-HMGCR myopathy. J Neuromuscul Dis 2018; 5: 11-20 doi:10.3233/JND-170282

[24] Griggs RC, Askanas V, DiMauro S et al. Inclusion body myositis and myopathies. Ann Neurol 1995; 38: 705-713 doi:10.1002/ana.410380504

[25] Pinal-Fernandez I, Casal-Dominguez M, Carrino JA et al. Thigh muscle MRI in immune-mediated necrotising myopathy: extensive oedema, early muscle damage and role of anti-SRP autoantibodies as a marker of severity. Ann Rheum Dis 2017; 76: 681-687 doi:10.1136/annrheumdis-2016210198

[26] Farrow M, Biglands ], Alfuraih AM et al. Novel Muscle imaging in inflammatory rheumatic diseases-a focus on ultrasound shear wave elastography and quantitative MRI. Front Med (Lausanne) 2020; 7: 434 doi:10.3389/ fmed.2020.00434

[27] Leeuwenberg KE, van Alfen N, Christopher-Stine L et al. UItrasound can differentiate inclusion body myositis from disease mimics. Muscle Nerve 2020; 61: 783-788 doi:10.1002/mus.26875

[28] Oddis CV, Aggarwal R. Treatment in myositis. Nat Rev Rheumatol 2018; 14: 279-289 doi:10.1038/ nrrheum.2018.42

Bibliografie

Neurologie up2date 2020; 3: 411-424

DOI 10.1055/a-1011-7010

ISSN 2511-3453

(c) 2020. Thieme. All rights reserved. Georg Thieme Verlag KG Rüdigerstraße 14, 70469 Stuttgart, Germany 


\section{Punkte sammeln auf CME.thieme.de}

Diese Fortbildungseinheit ist in der Regel 12 Monate online für die Teilnahme verfügbar.

Den genauen Einsendeschluss finden Sie unter https://cme.thieme.de.

Sollten Sie Fragen zur Online-Teilnahme haben, finden Sie unter https://cme.thieme.de/hilfe

eine ausführliche Anleitung. Wir wünschen viel Erfolg beim Beantworten

der Fragen!

Unter https://eref.thieme.de/CXDUFS8 oder über den QR-Code kommen Sie direkt zur Startseite des Wissenstests.

VNR 2760512020158722245

\section{Frage 1}

Welches Verteilungsmuster von Paresen ist für die Dermatomyositis (DM), die Polymyositis (PM) und die immun mediierte nekrotisierende Myopathie (IMNM) charakteristisch?

A asymmetrische proximale Paresen der Extremitäten und Schwäche der Stammmuskulatur

B Augenmuskelparesen, bilaterale Ptose und Facies myopathica

C symmetrische Paresen der Schulter-/Beckengürtel- und axialen Muskulatur

D Paresen der Knieextensoren/Vorfußheber und langen Fingerbeuger

E Paresen der Hüftbeuger, Kniestrecker und intrinsischen Handmuskulatur

\section{Frage 2}

Welche Aussage trifft für die Creatinkinase (CK) nicht zu?

A Eine starke CK-Erhöhung spricht bei der Myositis für eine ausgeprägte Muskelschädigung.

B Eine CK unter $1000 \mathrm{U} / \mathrm{I}$ und das Fehlen von Muskelschmerzen schließen eine Myositis aus.

C Die CK kann im chronischen Stadium einer Myositis abnehmen bzw. sich normalisieren.

D Eine Erhöhung der CK geht häufig mit einer Erhöhung der Laktatdehydrogenase und der Transaminasen einher.

E Die CK ist ein unspezifischer Marker einer Muskelschädigung, der auch ohne zugrundeliegende Myopathie erhöht sein kann.

\section{Frage 3}

Welche Hautmanifestationen sind für das Antisynthetase-

Syndrom typisch?
A Café-au-Lait-Flecken
B heliotropes Exanthem
C Mechanic's Hands
D Hautulzera
E Teleangiektasien

\section{Frage 4}

Eine Dermatomyositis mit TIF-Anti-TIF 1y-Antikörper zeigt eine häufige Assoziation mit...
A Raynaud Syndrom
B Arthralgien/Arthritiden.
C erhöhtem Tumorrisiko.
D interstitieller Lungenerkrankung.
E Nierenversagen.

\section{Frage 5}

Eine interstitielle Lungenerkrankung (ILD) kommt bei einer der genannten Formen einer Myositis nicht vor. Bei welcher?
A Antisynthetase-Syndrom
B Dermatomyositis mit MDA-5-Antikörpern
C Dermatomyositis ohne Antikörper
D Einschlusskörpermyositis
E Dermatomyositis mit EJ-Antikörpern

\section{Frage 6}

Welcher Befund ist bei der immun mediierten nekrotisierenden Myopathie (IMNM) nicht anzutreffen?
A ausgeprägte proximale Schwäche
B exzessive Erhöhung der Creatinkinase
$C$ axiale Schwäche und Dysphagie
D ausgeprägte distale Schwäche
E häufige Muskelschmerzen 


\section{Punkte sammeln auf CME.thieme.de}

Fortsetzung ...

\section{Frage 7}

Eine Statin-Exposition ist bei welcher Form einer Myositis typisch?
A Dermatomyositis mit Mi-2-Antikörpern
B immun mediierte nekrotisierende Myopathie mit SRP- Antikörpern
C immun mediierte nekrotisierende Myopathie mit HMGCR- Antikörpern
D Antisynthetase-Syndrom
E Dermatomyositis mit MDA-5-Antikörpern

\section{Frage 8}

Welches Verteilungsmuster der Paresen ist für die Einschlusskörpermyositis typisch?

A Schwäche für die Hüftbeugung und Nackenextension

B Schwäche für die Fingerendgliedbeugung und Knieextension

C Schwäche für die Hüftbeugung und Knieextension

D Schwäche für die Armabduktion und Vorfuß-/Großzehenhebung

E Schwäche für die Ellenbogenbeugung und Knieextension

\section{Frage 9}

Welche EMG-Veränderung ist für die akute Myositis nicht typisch?
A polyphasische Potenziale
B frühzeitige Rekrutierung
C scharf positive Wellen
D niedrige Entladungsrate
E erniedrigte Amplituden

\section{Frage 10}

Was ist ein typischer histologischer Befund einer Dermatomyositis?
A Fasernekrosen ohne entzündliches Infiltrat
B Faserinvasion durch CD8-positive T-Zellen
C perifaszikuläre Faseratrophien
D geränderte Vakuolen
E Proteineinschlüsse im Sarkoplasma 